\title{
French-English Cross-Linguistic Comparison and Diagnostic Impact of the AD-8 Dementia Screening Questionnaire in a Geriatric Assessment Clinic
}

\author{
Lisa Koski Haiqun Xie Susanna Konsztowicz Roslyn Tetteh \\ Research Institute of the McGill University Health Centre, Montreal, Que., Canada
}

\section{Key Words}

Language equivalence - Convergent validity diagnosis •

AD-8 screening questionnaire

\begin{abstract}
The AD-8 dementia screening questionnaire is a novel tool that allows clinicians to assess changes in cognitive function. This study examined psychometric properties of the test in French-speaking and English-speaking patients, and its impact on diagnostic practice in the geriatric assessment clinics of a university health centre. Data were extracted from the clinical database for all new patients screened for cognitive impairment 15 months before (historic control group) and 15 months after the introduction of the test (AD-8 group and concurrent control group). Analysis of differential item functioning revealed formal equivalence of the French- and English-language items, supporting the validity of the French version. Respondent type significantly influenced the total score on the AD-8. Concurrent validity with other cognitive screening tests was moderately high. Finally, among patients who did not present with pre-existing dementia, a higher proportion of dementia diagnoses was made in those administered the AD- 8 relative to the concurrent control group. Implications for clinical use of the AD-8 are discussed.

Copyright $\odot 2010$ S. Karger AG, Basel
\end{abstract}

\section{Introduction}

Screening for cognitive impairment is an important component of the geriatric clinical assessment. One of the criteria for diagnosing a progressive dementing disorder is that the cognitive impairment documented by clinician or informant report and/or abnormal scores on a performance-based screening test must represent a significant decline from a previous level of functioning. Performance-based screening tools do not allow us to measure decline based on a single administration of the test because they only measure current status. The newly developed Alzheimer's Dementia Questionnaire (AD-8) is one of several screening instruments designed to formalize the process of collecting data about the presence of a decline in cognitive functioning.

The AD-8 instructs the respondent to indicate whether there has been a change in the last several years in the ability to complete each of 8 tasks, e.g. remembering appointments. Although the method of collecting information is cross-sectional, the respondent must perform a comparison of current performance relative to past performance on the task. Endorsing at least 2 of 8 yes/no questions on the $\mathrm{AD}-8$ was found to be $83 \%$ accurate in classifying patients as demented, with $86 \%$ specificity and $74-85 \%$ sensitivity depending on the prevalence and se- 
verity of dementia in the sample [1]. The AD- 8 also shows good discrimination in patient samples that include nonamnestic dementias [2]. The AD- 8 has been validated in both English and Korean, showing strong internal consistency (Cronbach's $\alpha=0.84-0.88$ ), interrater reliability (0.82-0.89) and concurrent validity with the Clinical Dementia Rating scale and neuropsychological tests $[2,3]$.

The AD-8 was introduced into our geriatric cognitive assessment clinics in September 2007 as a part of the initial screening procedures for new referrals. Our clinical research team was interested in evaluating the appropriateness of this screening test for our clinic population, which is based in a different linguistic and health care context than the population sampled during the development of the test. As our clinic population is approximately one quarter French speaking, we translated the AD-8 into French, and the first objective of this study was to investigate the comparability of the English- and Frenchlanguage versions in our population.

Our second and third objectives were to examine the impact of respondent type (self-report vs. informant report) and of patient characteristics such as age, sex and educational level on $\mathrm{AD}-8$ performance. Objective 4 was to examine the convergent validity of the AD- 8 with respect to other screening tests, i.e. Mini Mental State Examination (MMSE) and the Older Americans Resource Scale (OARS).

Finally, objective 5 of this study was to determine the impact of the AD-8 screening test on diagnostic practice in the geriatric cognitive assessment clinic. All four existing publications on the $\mathrm{AD}-8$ came from a US centre that favours early diagnosis of dementia even in cases that would be considered questionable in other environments because of the absence of documented functional decline [4]. Thus, we hypothesized that the use of the AD- 8 questionnaire in our clinic might result in a shift in clinicians' diagnostic practices toward diagnosing dementia rather than mild cognitive impairment.

\section{Methods}

\section{Design}

A comparison of the English- and French-language versions of the $\mathrm{AD}-8$ was conducted in a cross-sectional study of the entire cohort of new patients who were administered the AD-8. A crosssectional design was also used to estimate the impact of the AD-8 screening test on diagnostic practice by comparing the relative frequency of dementia diagnoses in the group of new patients to whom the AD- 8 was administered with those of 2 control groups. Data for the study were extracted from a clinical database of geriatric cognitive assessments, described in detail below.

\section{Setting}

The study received ethical approval from the McGill University Health Centre Research Ethics Board. Data used in the study were obtained as part of a clinical epidemiology research project designed to characterize the nature and extent of cognitive impairment observed in individuals attending 2 outpatient geriatric assessment clinics. The clinics are located at 2 sites within a large urban university-based health centre: the Montreal General Hospital and the Royal Victoria Hospital. Clinic referrals come from family or specialist physicians, community services or from other services within the hospital. Referrals are made for general geriatric assessment or one of the subspecialties: cognitive disorders, pain, nutrition or incontinence. New referrals routinely undergo screening for cognitive impairment.

\section{Clinical Assessment Procedures}

Geriatric nurses or physicians administer screening tests to patients or informants during their first visit to the clinic. The basic set of screening tests includes the MMSE for cognitive assessment, the Barthel Index for assessment of functional ability in basic activities of daily living and the OARS for assessment of functional ability in instrumental activities of daily living. Following recommended practice guidelines [5], the Montreal Cognitive Assessment (MoCA) is administered when there are cognitive complaints without functional impairment, or as a secondstage screen when the MMSE score is $>25$. Starting in September 2007 , standard operating procedures for the clinic were modified to include the $\mathrm{AD}-8$ as one of the cognitive screening instruments administered to new referrals. The test is administered in either English or French according to the respondent's preference.

\section{The AD-8 Questionnaire}

The original English-language version of the AD-8 was downloaded from online supplemental materials provided by its authors [1]. The AD-8 was translated into French by a fully bilingual clinic nurse. The translated version was reviewed by two other bilingual members of the clinic team whose mother tongue was English or French and the final version was produced based on their feedback. For use in our clinic, additional space was added at the top of the form for recording the patient's demographic information and at the bottom to record the mode of administration (telephone/live) [2] and respondent (e.g. self, spouse, brother, daughter). Previous work indicates that patients can complete the AD-8 when no informant is available [6]. Unlike the original AD8 , we did not give the questionnaire to the respondent on a clipboard to fill out alone. Instead a clinic nurse asked the questions and recorded yes/no/don't know responses as well as any relevant comments made spontaneously by the respondent.

\section{Description of the Clinical Database}

The computerized database includes cognitive screening test data from 1999 to the present, with over 250 new entries annually since 2006, identified by medical record number and date. The database was designed to facilitate geriatric cognition research by capturing a level of detail that is atypical within a clinical setting. Included are: demographic information, diagnoses, itemized responses to behavioural screening tests and questionnaires and neuropsychological test results where available. New entries to the database are made by a research assistant and are triggered by cognitive testing or by a new diagnosis in all patients 
visiting the clinic each month. Diagnostic information comes from the physician's notes from the date on which screening tests are administered and is confirmed in multidisciplinary case conferences to ensure consistent application of diagnostic criteria.

\section{Diagnostic Criteria}

Primary diagnoses related to cognitive impairment are coded in the database. Dementia diagnoses are made clinically based on DSM-IV-TR criteria [7] and include Alzheimer's dementia, vascular dementia, frontotemporal dementia, Parkinson's dementia, Lewy body dementia and mixed dementias where 2 or more dementia types are specified. A diagnosis of cognitive impairment - no dementia (CIND) is made based on criteria used in the Canadian Study of Health and Aging, i.e. objective cognitive impairment in any domain that does not reach criteria for dementia, with specification of subtypes as amnestic single-domain, amnestic multidomain or non-amnestic, and aetiology where known. When the diagnosis of dementia versus CIND is questionable, the term 'CIND vs. dementia' is used. Cases for which cognitive impairment is deemed to result primarily from a mood, anxiety or psychotic disorder are classified under the category 'psychiatric cognitive impairment'. The category of 'cognitive impairment not yet diagnosed' was used in cases where there was evidence of cognitive impairment but where neither the severity nor the aetiology of the impairment had yet been determined. 'Cognitively normal' was used for those patients who presented without cognitive functional decline and who screened negative for cognitive impairment. A complete list of diagnoses is shown in table 1.

\section{Study Groups}

We extracted demographic, clinical and diagnostic data for all new admissions to the clinics who underwent cognitive screening with the MMSE and/or MoCA, beginning when the AD-8 was introduced (from September 10, 2007, to December 11, 2008). Those patients who were administered the AD- 8 comprise the $A D-8$ group (AD-8, $\mathrm{n}=146)$. Those patients who were not administered the $\mathrm{AD}-8$ comprise the concurrent control group $(\mathrm{n}=156)$. In addition, we created a historic control group by retroactively extracting cases from between June 8, 2006, and September 9, 2007 , in order to obtain a group of comparable size to the concurrent groups $(n=171)$. A flowchart of the procedures for group selection is shown in figure 1 . For analyses of the psychometric properties of the AD-8 (objectives 1-4), the entire AD- 8 group was retained.

The goal of objective 5 was to evaluate whether knowledge of AD-8 test results would influence the clinical diagnosis for patients in whom dementia was not already clearly present. Therefore, we only retained for further analysis those patients who obtained an MMSE score $\geq 20 / 30$, which corresponds to the cut-off for the lower 5th percentile among the oldest population of community-dwelling Canadian elderly subjects with at least elementary school education [8]. MMSE data were missing for 12 cases ( $2.5 \%$ of the sample). Of these, 10 cases were retained because they had a MoCA score $>18$, a cut-off that would include $>84 \%$ of the distribution of people with mild cognitive impairment based on data presented by Nasreddine et al. [5]. One additional case was retained because an MMSE done at a follow-up visit 4 months later was $26 / 30$. The last case was excluded because no screening test data were available with the exception of the AD-8.

AD-8 French-Language Translation and Diagnostic Impact
Table 1. Classification scheme for diagnostic categories

\begin{tabular}{ll}
\hline Classification & Diagnoses \\
\hline Dementia & $\begin{array}{l}\text { Alzheimer's dementia } \\
\text { Vascular dementia } \\
\text { Frontotemporal dementia } \\
\text { Parkinson's dementia } \\
\text { Lewy body dementia } \\
\text { Mixed dementia (types specified) }\end{array}$ \\
\hline Dementia vs. CIND & $\begin{array}{l}\text { Diagnosis of dementia vs. CIND } \\
\text { is questionable }\end{array}$ \\
\hline No dementia & CIND \\
& Cognitively normal \\
\hline Other & Psychiatric cognitive impairment \\
& Cognitive impairment not yet diagnosed
\end{tabular}

See text for definitions and diagnostic criteria.

\section{Extraction from the Database}

The following information was extracted from the clinical database: clinic site (Montreal General Hospital or Royal Victoria Hospital), patient sex, patient years of education, language of test, patient date of birth, date of test, patient diagnosis at time of test and itemized test scores for the $\mathrm{AD}-8$. The language of test was classified as English, French or other (most commonly Italian, Spanish or Cantonese). When the AD- 8 had been administered we also recorded the type of respondent (objective 2) using the classification system 'self' (only the patient was interviewed), 'peer' (patient's spouse or sibling was interviewed), 'child' (patient's child was interviewed) or 'other' (patient's friend or caregiver was interviewed). For objective 3 , years of education were coded as $<13$ or $\geq 13$ years, and age was coded as $<80$ or $\geq 80$ years. Also extracted were the patient's total scores out of 30 on the MMSE and the total score out of 14 on the OARS (objective 4).

\section{Diagnostic Coding}

To evaluate the impact of the AD-8 on frequency of dementia diagnosis (objective 5), each patient's diagnosis was coded into the general categories 'dementia', 'no dementia' (CIND or cognitively normal), 'CIND vs. dementia' or 'other'. The 'other' category included the diagnoses 'cognitive impairment not yet diagnosed' and 'psychiatric cognitive impairment' (table 1).

\section{Data Analyses}

Test of French-Language Equivalence (Objective 1). In the AD-8 group, the English-speaking and French-speaking subgroups were compared for differences in the distributions of age, sex, years of education and test site. To evaluate the similarity of the French-language and English-language AD-8, we first conducted a 1-way ANOVA to test for bias in the total score as a function of language. Next we conducted a more rigorous test of the equivalence of the 2 versions at the individual-item level in the context of a Rasch analysis of the AD-8 data. Using RUMM2020 software (RUMM Laboratory Pty Ltd., 2007), we tested for differential item 


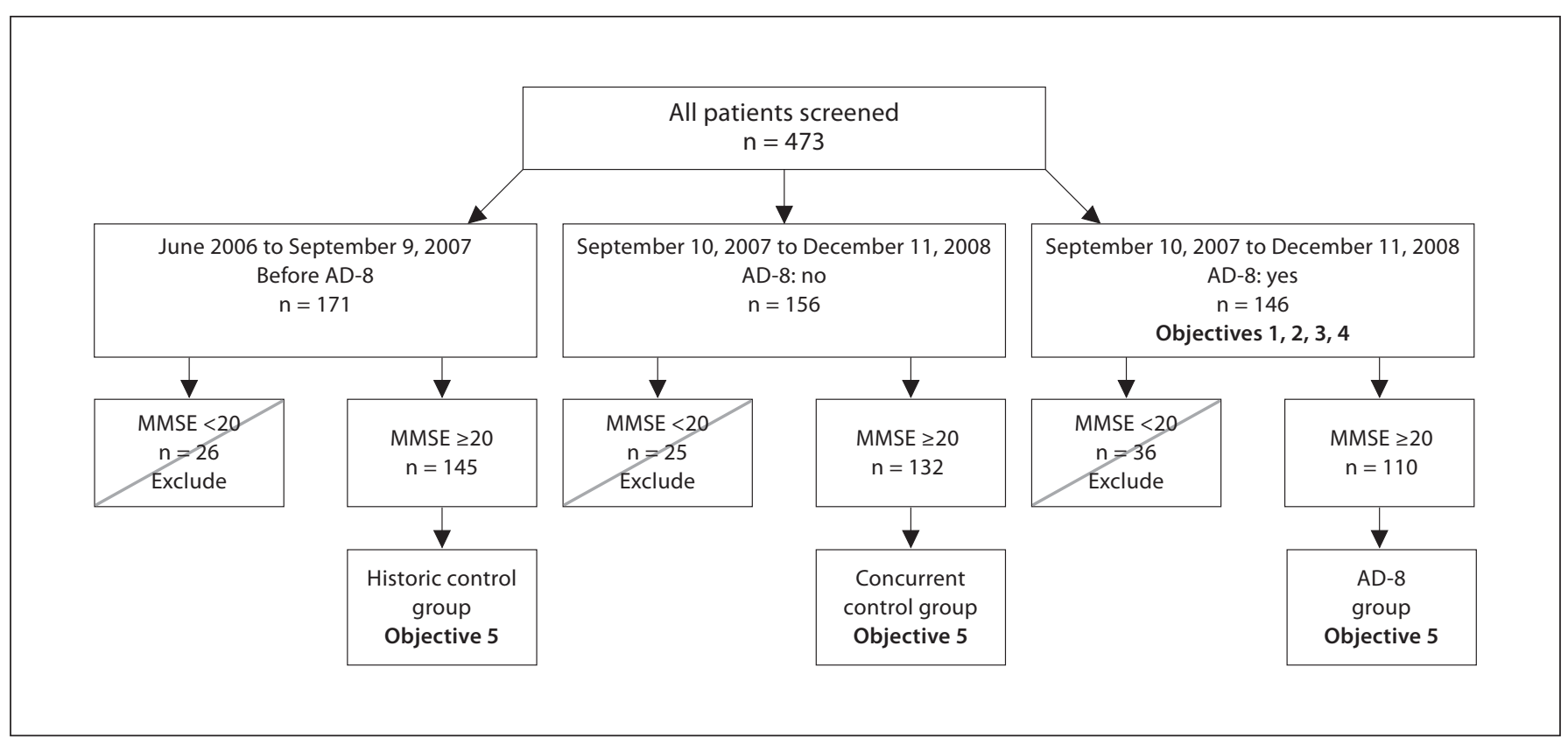

Fig. 1. Selection of cases comprising the groups included for each study objective.

functioning related to test language with a 2-way ANOVA (3 disability levels by 2 languages) and a cut-off p value of 0.05 .

Influence of Respondent Type (Objective 2) and Patient Characteristics (Objective 3). The same differential item functioning approach was also used to examine the influence of site, age, sex, education and type of respondent (self, peer, child or other) on both the total score and on the probability of responding yes versus no to individual items. For these analyses, we used a cut-off criterion of $\mathrm{p}=0.05$, uncorrected for multiple comparisons.

Convergent Validity (Objective 4). We calculated Spearman rank correlations between the total score on the AD-8 and the total scores on (1) the MMSE and (2) the OARS Instrumental Activities of Daily Living scale.

Impact of AD-8 on Diagnostic Practice (Objective 5). Frequencies, means and standard deviations were used to characterize the 3 groups on demographic variables (age, sex, years of education and language of testing) and MMSE total score. Over half of the patients screened after the introduction of the $\mathrm{AD}-8$ were not administered this test. Therefore patient characteristics were examined as potential sources of bias that might have guided the selection of those for whom the AD-8 was administered versus not administered. To examine potential differences between the 3 groups, we used the $\chi^{2}$ test for demographic categorical variables and single-factor analysis of variance on the MMSE. For these analyses, we used a cut-off for significance of $p=0.05$, uncorrected for multiple comparisons. We used separate $\chi^{2}$ tests with a cut-off for significance of $\mathrm{p}=0.05$ (uncorrected) to compare the frequencies of diagnostic classifications (4 levels) in the $\mathrm{AD}-8$ group versus the concurrent control group, and in the $\mathrm{AD}-8$ group versus the historic control group.

\section{Results}

\section{Test of French-Language Equivalence (Objective 1)}

Demographic and clinical characteristics of the English- and French-language subgroups of the AD-8 group are shown in table 2 . When compared with the English-language subgroup, the French-language subgroup obtained significantly higher scores on the MMSE but also showed a non-significant trend toward a higher proportion of patients with 13 or more years of education. The subgroups did not differ in terms of their test site, sex, age or respondent type distributions, by their total scores on the AD-8 or OARS, or by the proportion of patients obtaining all 'yes' or all 'no' responses.

In the English-language subgroup, the diagnostic classification was normal for 2 , CIND for 18 , CIND vs. dementia for 3, dementia (all kinds) for 57, psychiatric for 10 and cognitive impairment not yet diagnosed for 8 . In the French-language subgroup, the diagnostic classification was normal for 2, CIND for 8 , CIND vs. dementia for 2, dementia (all kinds) for 21, psychiatric for 5 and cognitive impairment not yet diagnosed for 3 .

Analysis of differential item functioning was completed for all patients who had a mix of both 'yes' and 'no' responses on the $\mathrm{AD}-8$. One hundred thirty-four respondents (93\% of the sample) answered 'yes' to at least 1 ques- 
tion on the $\mathrm{AD}-8$. One hundred fourteen respondents (79\% of the sample) answered 'no' to at least 1 question on the AD-8. Data from the remaining patients had to be excluded from the analysis of differential item functioning because they provide no information about the relative severity of impairment represented by the individual items. Table 2 displays the distribution of responses for and the degree of cognitive decline represented by each AD-8 item. The AD-8 total score did not differ significantly between the English- and French-language subgroups $\left(\mathrm{F}_{2,143}=1.1, \mathrm{p}=0.34\right)$. Moreover, tests for differential item functioning revealed no differences between the two languages in how respondents answered individual items (neither uniform nor non-uniform differential item functioning, $\mathrm{p}>0.10$ for all items).

\section{Influence of Respondent Type (Objective 2)}

Overall scores were highest for 'other' respondents, followed by 'child' respondents, then 'spouse' informants and finally scores were lowest for 'self-respondents' $(\mathrm{F}=$ $8.06, \mathrm{p}=0.00006)$. There was no evidence for differential item functioning as a function of respondent type $(\mathrm{p}>$ 0.05).

\section{Influence of Patient Characteristics (Objective 3)}

Scores were higher, representing greater cognitive decline, for older ( $\geq 80$ years) versus younger patients $(<80$ years; $\mathrm{F}=3.87, \mathrm{p}=0.05)$. AD- 8 scores did not differ by sex or education of the patient. There was no evidence for differential item functioning as a function of age, sex or education (all $\mathrm{p}>0.05$ ).

\section{Convergent Validity (Objective 4)}

As the number of cognitive tasks showing functional decline on the AD-8 increased, performance on the MMSE declined $(\mathrm{r}=-0.56, \mathrm{p}<0.0001)$ and functional ability in instrumental activities of daily living (OARS) declined $(\mathrm{r}=-0.59, \mathrm{p}<0.0001)$.

\section{Comparisons of Group Characteristics and Impact of}

AD-8 on Diagnostic Practice (Objective 5)

Demographic and diagnostic data for the 3 groups are presented in table 3 , along with the results of the statistical analysis comparing the groups on demographic and clinical variables. We observed a difference between the 2 clinic sites, with the Royal Victoria Hospital administering the $\mathrm{AD}-8$ to a greater proportion of patients than the Montreal General Hospital (55 vs. $36 \%, \chi^{2}=8.9, \mathrm{p}=$ $0.003)$. The groups did not differ in terms of their distributions for the variables sex, age, education or MMSE
Table 2. Characteristics of English- and French-speaking AD-8 groups $^{\text {a }}$ : frequency distributions and means \pm standard deviations

\begin{tabular}{|c|c|c|c|}
\hline & $\begin{array}{l}\text { Anglophone } \\
\text { AD-8 group } \\
(n=103)\end{array}$ & $\begin{array}{l}\text { Francophone } \\
\text { AD-8 group } \\
(\mathrm{n}=41)\end{array}$ & $\mathrm{p}$ value $\mathrm{e}^{\mathrm{b}}$ \\
\hline \multicolumn{4}{|l|}{ Clinic site } \\
\hline MGH & 44 & 18 & \multirow[t]{2}{*}{0.90} \\
\hline $\mathrm{RVH}$ & 59 & 23 & \\
\hline \multicolumn{4}{|l|}{ Sex } \\
\hline Female & 62 & 23 & \multirow[t]{2}{*}{0.65} \\
\hline Male & 41 & 18 & \\
\hline \multicolumn{4}{|l|}{ Age, years } \\
\hline mean $\pm S D$ & $80.5 \pm 6.6$ & $78.9 \pm 7.1$ & 0.19 \\
\hline$<80$ & 44 & 19 & \multirow[t]{2}{*}{0.87} \\
\hline$\geq 80$ & 59 & 24 & \\
\hline \multicolumn{4}{|l|}{ Education, years } \\
\hline$\geq 13$ & 20 & 14 & \multirow[t]{3}{*}{0.08} \\
\hline$<13$ & 66 & 22 & \\
\hline Missing & 17 & 5 & \\
\hline \multicolumn{4}{|l|}{ Respondent } \\
\hline Self & 30 & 11 & \multirow[t]{5}{*}{0.14} \\
\hline Peer & 13 & 12 & \\
\hline Child & 52 & 17 & \\
\hline Other & 5 & 1 & \\
\hline Missing & 3 & 0 & \\
\hline \multicolumn{4}{|l|}{ Test scores } \\
\hline MMSE & $21.7 \pm 6.2^{c}$ & $24.1 \pm 5.1$ & $0.02^{*}$ \\
\hline $\mathrm{MoCA}$ & $18.7 \pm 4.9$ & $19.4 \pm 5.5$ & 0.53 \\
\hline OARS & $8.4 \pm 4.0^{c}$ & $9.2 \pm 3.9^{c}$ & 0.25 \\
\hline AD-8 & $4.7 \pm 2.7$ & $4.0 \pm 2.5$ & 0.19 \\
\hline \multicolumn{4}{|l|}{ AD-8 extremes } \\
\hline All 'yes' & 26 & 4 & \multirow[t]{2}{*}{0.62} \\
\hline All 'no' & 8 & 2 & \\
\hline
\end{tabular}

MGH $=$ Montreal General Hospital; RVH = Royal Victoria Hospital.

${ }^{*} \mathrm{p}<0.05$ : statistically significant, uncorrected for multiple comparisons.

a The test was translated into Italian for 2 patients, reducing the total number to 144 for this table.

${ }^{\mathrm{b}} \mathrm{p}$ values correspond to the significance of the test comparing the two subgroups using $\chi^{2}$ or Fisher's exact test for categorical variables or a 2-tailed independent $t$ test for continuous variables.

${ }^{\mathrm{c}}$ Missing data for 2 English MMSE (1.3\%) and 9 OARS assessments (6.3\%, 8 English, 1 French).

score. This allows us to rule out these known predictors of dementia as potential confounding variables in our analysis.

The diagnostic frequency distribution in the AD-8 group differed significantly from that of the concurrent control group $\left(\chi^{2}=9.54, \mathrm{p}=0.02\right)$ but not the historic 


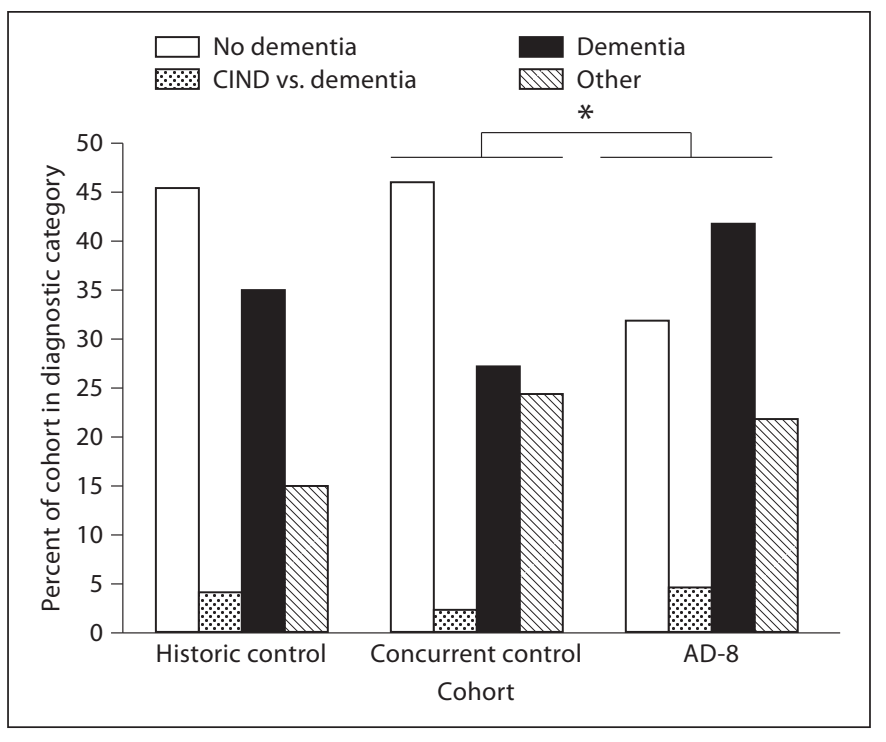

Fig. 2. Diagnostic frequency distribution for the 3 groups. ${ }^{*} \mathrm{p}=$ 0.02 for the $\chi^{2}$ test comparing diagnostic frequency distributions of the AD-8 group vs. the concurrent control group.

control group $\left(\chi^{2}=5.25, p=0.16\right)$. Figure 2 illustrates the differences in diagnostic frequency distributions across groups. Examination of the observed and expected frequencies for each cell showed the largest deviations from expected values in the no-dementia and dementia categories: no dementia was diagnosed with greater than expected frequency in the concurrent control group and with less than expected frequency in the AD-8 group. Dementia was diagnosed with greater than expected frequency in the AD-8 group and with less than expected frequency in the concurrent control group. The other 2 diagnostic categories contributed little to the significant overall $\chi^{2}$ statistic. We conducted post-hoc pairwise comparisons; however, none of the comparisons were significant, consistent with the interpretation that differences in proportions were caused by complex contrasts [9]. A test of the simple $2 \times 2$ table with 1 degree of freedom containing only the categories 'no dementia' and 'dementia' yielded a $\chi^{2}$ statistic of $6.88, \mathrm{p}=0.009$.

For the $\mathrm{AD}-8$ group, 10 respondents $(6.9 \%$ of the sample) answered 'no' or 'not sure' to all questions on the $\mathrm{AD}$ 8 . None of the corresponding patients were subsequently diagnosed as having dementia. Thirty respondents (20.8\% of the sample) answered 'yes' or 'not sure' to all questions on the AD-8. Twenty-eight of these were diagnosed as having dementia.

Two patients who did not reach the diagnostic cut-off of 2 'yes' responses on the $\mathrm{AD}-8$ were diagnosed as having
Table 3. Distribution of characteristics in the 3 groups, as a percentage of the total number in each group

\begin{tabular}{|c|c|c|c|c|c|}
\hline & $\mathrm{HC}$ & $\mathrm{CC}$ & $\mathrm{AD}-8$ & $\begin{array}{l}\mathrm{p} \text { value } \\
\mathrm{HC} \text { vs. } \\
\mathrm{AD}-8\end{array}$ & $\begin{array}{l}p \text { value } \\
\text { CC vs. } \\
\text { AD- } 8\end{array}$ \\
\hline Number & 145 & 132 & 110 & & \\
\hline \multicolumn{6}{|l|}{ Clinic site } \\
\hline MGH & 48 & 60 & 41 & 0.24 & 0.003 \\
\hline RVH & 52 & 40 & 59 & & \\
\hline \multicolumn{6}{|l|}{ Sex } \\
\hline Female & 50 & 58 & 55 & 0.51 & 0.55 \\
\hline Male & 50 & 42 & 45 & & \\
\hline \multicolumn{6}{|l|}{ Age, years } \\
\hline Mean $\pm S D$ & $81 \pm 6$ & $81 \pm 7$ & $80 \pm 7$ & 0.15 & 0.15 \\
\hline$<80$ & 38 & 44 & 45 & 0.23 & 0.81 \\
\hline$\geq 80$ & 62 & 56 & 55 & & \\
\hline \multicolumn{6}{|l|}{ Education, years } \\
\hline$<13$ & 34 & 29 & 29 & $0.11^{\mathrm{a}}$ & $0.46^{\mathrm{a}}$ \\
\hline$\geq 13$ & 42 & 45 & 57 & & \\
\hline Missing & 24 & 26 & 14 & & \\
\hline \multicolumn{6}{|l|}{ MMSE } \\
\hline Mean $\pm S D$ & $25.9 \pm 2.6$ & $25.6 \pm 2.9$ & $25.4 \pm 2.7$ & 0.12 & 0.64 \\
\hline
\end{tabular}

$\mathrm{HC}=$ Historic control; $\mathrm{CC}=$ concurrent control; $\mathrm{MGH}=$ Montreal General Hospital; RVH = Royal Victoria Hospital; $\mathrm{SD}=$ standard deviation; $\mathrm{HC}$ vs. $\mathrm{AD}-8=\mathrm{p}$ value for $2 \times 2 \chi^{2}$ or $\mathrm{t}$ statistic comparing historic control and AD- 8 groups on different levels of the factor of interest; $\mathrm{CC}$ vs. $\mathrm{AD}-8=\mathrm{p}$ value for $2 \times 2 \chi^{2}$ or t statistic comparing concurrent control and $\mathrm{AD}-8$ groups on different levels of the factor of interest.

a Analysis excludes missing data.

dementia (both scored $1 / 8$ as per child respondent). Of the 37 cases diagnosed as CIND or normal, 24 would have been classified as demented if diagnosis were made solely on the basis of the recommended cut-off of 1 'yes' response for a self-respondent or 2 'yes' responses for an informant-respondent. The AD-8 scores for the CIND group spanned the full range from 0 to 8 .

\section{Discussion}

The present findings constitute the first evidence from Canadian geriatric outpatient assessment clinics on the use and clinical impact of the AD-8 screening test. We have provided support for the validity of a French-language translation of the AD-8 Dementia Screening Questionnaire by demonstrating its comparability with the original English-language version in terms of the response properties of its items in a clinical geriatric sample 
Table 4. Response frequency distributions and Rasch modelled item location for each AD-8 item

\begin{tabular}{|c|c|c|c|c|c|}
\hline \multicolumn{2}{|c|}{ Item } & \multirow{2}{*}{$\begin{array}{l}\text { No } \\
59\end{array}$} & \multirow{2}{*}{$\begin{array}{c}\text { Yes } \\
45\end{array}$} & \multirow{2}{*}{$\begin{array}{c}\text { Not } \\
\text { sure }\end{array}$} & \multirow{2}{*}{$\begin{array}{l}\begin{array}{l}\text { Item } \\
\text { location }\end{array} \\
+0.46\end{array}$} \\
\hline$(1)$ & $\begin{array}{l}\text { Problèmes de jugement (p. ex. difficulté à prendre des décisions, } \\
\text { mauvaises décisions financières, trouble de la pensée) }\end{array}$ & & & & \\
\hline$(2)$ & Moins d’intérêt dans ses loisirs & 68 & 35 & 1 & +0.77 \\
\hline$(3)$ & Se répète souvent (questions, histoires ou déclarations) & 39 & 63 & 2 & -0.63 \\
\hline$(4)$ & $\begin{array}{l}\text { Difficulté à apprendre comment utiliser un nouvel appareil } \\
\text { (p. ex. micro-ondes, ordinateur, magnétoscope) }\end{array}$ & 56 & 37 & 11 & +0.84 \\
\hline$(5)$ & Oubli du mois/année & 66 & 35 & 3 & +0.87 \\
\hline$(6)$ & $\begin{array}{l}\text { Difficulté à gérer ses finances (p. ex. impôts, payer les comptes, } \\
\text { verifier les relevés de compte bancaires) }\end{array}$ & 42 & 58 & 4 & -0.44 \\
\hline$(7)$ & Difficulté à se souvenir de ses rendez-vous & 41 & 61 & 2 & -0.44 \\
\hline$(8)$ & Problèmes quotidiens avec sa mémoire/raisonnement & 24 & 78 & 2 & -1.43 \\
\hline
\end{tabular}

French version shown here. For English version of questions, see Galvin et al. [1]. Item location = Severity of decline represented by the item in units of logits, as calibrated on an interval scale by fitting to a normal distribution of cognitive functional decline (mean $=0$, standard deviation $=1$ ). Locations $>0$ indicate those items with a lower than average probability of being endorsed ('yes'), whereas locations $<0$ indicate those items with a greater than average probability of being endorsed. Endorsing item 8 represents the lowest level of decline, whereas endorsing item 5 represents the highest level of decline.

(objective 1). Although the 2 versions were compared between 2 different groups in this convenience sample, the analysis of differential item functioning lends strength to our conclusions about their functional equivalence. The French-language version performed similarly to the original English-language version, not just in terms of the total score, but also at the level of individual item responses. In other words, the magnitude of cognitive dysfunction established by the questions, individually and collectively, is comparable across the 2 versions.

Our Rasch analysis demonstrated the incidental finding that the $\mathrm{AD}-8$ is reasonably well targeted at the mean level of cognitive decline seen in our geriatric outpatient assessment clinics. The mean decline of +0.397 logits observed in the patients is only slightly above the zero level representing the mean decline indexed by the test items. However, the items measure cognitive decline within a relatively narrow range of the full spectrum of decline observed in the patient group (table 4). A full $24 \%$ of the group screened responded in the extremes - either all 'yes' responses or all 'no' responses. Thus, the use of the $\mathrm{AD}-8$ as a quantitative measure of cognitive decline is restricted to a limited group of patients. However, this property of greater precision close to a cut point is a preferred characteristic in a test that is intended to screen for cognitive decline by accurately classifying people as demented versus not demented. The range of cognitive decline assessed by the AD-8 is comparable to that assessed by the IQCODE, a commonly used but lengthier informant report scale with multiple response options for measuring cognitive decline [10]. There is substantial overlap in the content of the questions, suggesting that they may measure the construct of cognitive decline similarly.

We have also provided further evidence of the impact of respondent type on the total AD-8 score (objective 2). Scores were lower, reflecting less cognitive decline, when responses were provided by the patients themselves than when they were provided by a spouse, sibling or other family member. Peer respondents, in turn, reported less cognitive decline than offspring respondents. These findings beg the question of who is a more accurate respondent. There is ample evidence that patients with dementia may be unaware of the degree of their own impairment, making them less accurate respondents. This has been demonstrated for the AD-8 test [6] as well as for other self-report instruments [11, 12]. However, patients with mild cognitive impairment do not show the same lack of awareness of cognitive problems as those with dementia and may even be more sensitive than informants to changes in memory ability [12-14]. 
The impact of informant-patient relationship on ratings of cognitive ability for patients with mild cognitive impairment or dementia is not yet well understood. In previous work on this topic, rater accuracy, as indexed by correlations with performance-based cognitive measures, was found to be highest in respondents who were married to, living with or in frequent contact with the patient [15]. However, a recent study found cross-cultural differences in the effect of respondent-patient relationship [16]. Spouses in an African-American sample gave higher ratings of cognitive ability and were less accurate than other respondents, whereas spouses in a Nigerian sample gave lower ratings of cognitive ability than siblings but were more accurate. The latter study included a follow-up component in which cognitive decline, as measured by a change in informant ratings over 5 years, was not affected by informant-patient relationship.

We are limited in our ability to address the issue of accuracy in our informant respondents not only by the small sample size, but also by the mismatch in what is assessed by the two tests used in the current study. The MMSE assesses current performance, whereas the AD-8 asks whether there has been a decline from a previous level of ability, thus only modest correlations would be expected between scores on the two measures. Nevertheless, we did observe differences in correlations between AD-8 ratings and MMSE total scores by respondent type, with lower correlations for the spouse respondents $(-0.36)$ than for the child or other respondents. Interestingly, self-respondents were as accurate $(-0.56)$ as their children $(-0.50)$ and others $(-0.60)$. While contributing factors are far from clear, clinicians do need to be aware of the potential impact of respondent type when interpreting the results of the $\mathrm{AD}-8$ test. Two patients with the same true degree of cognitive decline may obtain different scores on the test if it is completed by different respondent types.

Of the patient characteristics evaluated, only age was found to affect the AD-8 score (objective 3). Not surprisingly, older patients tended to show decline on more tasks than did younger patients; however, the way respondents answered the individual items did not change as a function of age. Moreover, we did not observe any impact of patient age or educational level on the responses to the $\mathrm{AD}-8$. The relative insensitivity of the $\mathrm{AD}-8$ to a patient's educational level represents an advantage over performance-based tests of cognitive ability such as the MMSE and the MoCA, where a higher level of education facilitates higher scores [17-20] and thereby may obscure the early signs of dementia. Moreover, the AD- 8 focus on change from a previous functional level allows respondents to assess function relative to the patient's premorbid level rather than to an absolute standard of cognitive functioning.

Previous work by others on construct validity found mild-to-moderate negative correlations between total AD- 8 scores and MMSE scores $(r=-0.41)$ [2]. The results of the current study replicate those findings $(r=-0.56)$, providing further support for the convergent validity of informant or self-report as an indicator of cognitive disability secondary to dementia (objective 4). That the correlations are not stronger suggests that the $\mathrm{AD}-8$ may provide information about cognitive decline that is independent of that provided by the MMSE and thus may be a useful complement to the initial baseline evaluation of cognitive functioning.

A novel finding from this study is the moderate correlation between the AD- 8 and the OARS. There is some overlap in items for these two tests (i.e. difficulty with finances) but both scales consist of items intended to capture the construct of functional disability in cognitive tasks. One distinction between the OARS and the AD-8 is that the former may be influenced by disability that is caused by physical impairment as well as cognitive impairment. A second distinction is that the OARS is intended to measure the degree of current disability in cognitive functioning, whereas the $\mathrm{AD}-8$ is intended to measure change in cognitive functioning relative to a previous level of ability, or increased difficulty in performing tasks that does not necessarily entail functional dependence. As such, the AD- 8 may be more sensitive than the OARS to subtle changes in functioning, although the design of the current study does not allow us to test this hypothesis.

Finally, we have presented evidence for the diagnostic impact of the AD-8 (objective 5). Our data suggest that use of the AD-8 influences diagnostic practice, resulting in a higher proportion of dementia diagnoses in the group that was administered the test. The change relative to the historic control group was only a trend and did not reach criterion for statistical significance. However, a significant shift in the proportion of dementia diagnoses was observed when comparing the $\mathrm{AD}-8$ group with the concurrent control group.

In the absence of the $\mathrm{AD}-8$ screening test, the proportion of new patients receiving a diagnosis of cognitively normal or cognitive impairment not meeting criteria for dementia was $66 \%$ (historic control) or $61 \%$ (concurrent control). This proportion dropped to $35 \%$ when the AD-8 was used, i.e. $70 \%$ of the historic base rate for this diag- 
nostic category and $69 \%$ of the rate for concurrent controls. When the AD- 8 screening test is used, the rate at which patients are given a dementia diagnosis increases by $19 \%$ over base rates (historic control) and by $53 \%$ relative to current patients who are not administered the AD-8 at intake (concurrent control). The estimated annual number of new patients to undergo cognitive screening is 194 based on the current period of study (concurrent control $+\mathrm{AD}-8$ groups). Using the available data, we calculate that systematic use of the AD-8 for all new patients would result in an increase from 53 to 81 new patients receiving a dementia diagnosis annually.

\section{Limitations}

These results were obtained in a clinical setting in which English-speaking and French-speaking respondents are sociodemographically similar and share access to the same public health care system. The results of this study may not be generalized to other French-speaking cultures or to societies that differ significantly from urban North American populations. The study draws on data from a convenience sample of patients evaluated in the course of normal clinical practice and therefore did not include random assignment to the $\mathrm{AD}-8$ versus concurrent control groups. A limitation of this approach is that we have not controlled for unknown factors that might influence whether or not a patient was administered the AD-8 after its implementation. Nevertheless, we found that the groups did not differ on several important variables that might have confounded interpretation, such as patient age, sex, educational level and MMSE score.

Since insight is impaired in dementia, it is reasonable to expect that dementia will be underdiagnosed when there is no independent source of information. In our study, the proportion of dementia diagnoses was indeed higher in the $\mathrm{AD}-8$ group when the test was completed by an informant (62\% of diagnoses) versus self-reporting (28\% of diagnoses). Unfortunately, we do not have data on the source of the information used to make a clinical diagnosis in patients who did not have an AD-8 administered and, therefore, cannot determine whether the impact of $\mathrm{AD}-8$ use on diagnosis is influenced by the source of information. However, we know from previous work by others that a self-completed $\mathrm{AD}-8$ can discriminate between demented and non-demented cases when a lower cut-off of $1 / 8$ is used [6]. Those findings would suggest that administering the $\mathrm{AD}-8$ directly to a patient is better than not administering it at all. Our results suggest that dementia may be underdiagnosed when a test like the
AD-8 is not administered, although we cannot state this conclusively in the absence of an independent definitive test for dementia.

\section{Conclusions}

Although assessment of a decline in cognitive functioning is part of the usual clinical interview with the patient and family members, the use of specific questions may encourage more rigorous evaluation of cognitive decline, as well as cut-offs for concluding that decline is clinically significant. Since diagnoses were made in full knowledge of screening test results, this study was not designed to assess diagnostic accuracy, which has been demonstrated previously.

A change in diagnostic practice has implications for the management of geriatric clinics. Higher numbers of dementia diagnoses may lead to an increased number of clinic visits, and a more frequent follow-up may be required to monitor the effects of newly initiated pharmacotherapies. This impact on clinic resources must be weighed against the possibility of earlier detection and treatment of dementia, and hence improved patient care, by the use of a standardized screening instrument.

\section{Acknowledgements}

Special thanks go to Guylaine Bachand, RN, for drafting the French translation of the AD-8 and to Gary Inglis, MD, for feedback and comments on the translation. We gratefully acknowledge the work of the nurses and physicians of the Geriatric Outpatient Assessment Clinics of the Montreal General Hospital and the Royal Victoria Hospital, who administered the screening tests and contributed to the determination of diagnostic classification in our clinic population. This study was funded by a Hutchison Award in Geriatric Medicine through the Research Institute of the McGill University Health Centre.

References

Dement Geriatr Cogn Disord 2010;29:265-274
1 Galvin JE, Roe CM, Powlishta KK, Coats MA, Muich SJ, Grant E, Miller JP, Storandt M, Morris JC: The AD8: a brief informant interview to detect dementia. Neurology 2005;65:559-564.

2 Galvin JE, Roe CM, Xiong C, Morris JC: Validity and reliability of the AD8 informant interview in dementia. Neurology 2006;67: 1942-1948.

3 Ryu HJ, Kim HJ, Han SH: Validity and reliability of the Korean version of the AD8 informant interview (K-AD8) in dementia. Alzheimer Dis Assoc Disord 2009;23:371376. 
4 Morris JC, Storandt M, Miller JP, McKeel DW, Price JL, Rubin EH, Berg L: Mild cognitive impairment represents early-stage Alzheimer disease. Arch Neurol 2001;58:397405.

5 Nasreddine ZS, Phillips NA, Bedirian V, Charbonneau S, Whitehead V, Collin I, Cummings JL, Chertkow H: The Montreal Cognitive Assessment, MoCA: a brief screening tool for mild cognitive impairment. J Am Geriatr Soc 2005;53:695-699.

-6 Galvin JE, Roe CM, Coats MA, Morris JC: Patient's rating of cognitive ability: using the AD8, a brief informant interview, as a selfrating tool to detect dementia. Arch Neurol 2007;64:725-730.

7 Association AP: Diagnostic and Statistical Manual of Mental Disorders, ed 4, text revision. Washington, American Psychiatric Association, 2000.

$>8$ Iverson GL: Interpretation of Mini-Mental State Examination scores in communitydwelling elderly and geriatric neuropsychiatry patients. Int J Geriatr Psychiatry 1998;13: 661-666.

-9 Cox MK, Key CH: Post hoc pair-wise comparisons for the chi-square test of homogeneity of proportions. Educ Psychol Meas 1993;53:951-962.
10 Butt Z: Sensitivity of the informant questionnaire on cognitive decline: an application of item response theory. Neuropsychol Dev Cogn B Aging Neuropsychol Cogn 2008;15:642-655.

11 Farias ST, Mungas D, Jagust W: Degree of discrepancy between self and other-reported everyday functioning by cognitive status: dementia, mild cognitive impairment, and healthy elders. Int J Geriatr Psychiatry 2005; 20:827-834.

12 Kalbe E, Salmon E, Perani D, Holthoff V, Sorbi S, Elsner A, Weisenbach S, Brand M, Lenz O, Kessler J, Luedecke S, Ortelli P, Herholz K: Anosognosia in very mild Alzheimer's disease but not in mild cognitive impairment. Dement Geriatr Cogn Disord 2005;19:349-356.

13 Cook S, Marsiske M: Subjective memory beliefs and cognitive performance in normal and mildly impaired older adults. Aging Ment Health 2006;10:413-423.

14 Reisberg B, Gauthier S: Current evidence for subjective cognitive impairment (SCI) as the pre-mild cognitive impairment (MCI) stage of subsequently manifest Alzheimer's disease. Int Psychogeriatr 2008;20:1-16.

15 Cacchione PZ, Powlishta KK, Grant EA Buckles VD, Morris JC: Accuracy of collateral source reports in very mild to mild dementia of the Alzheimer type. J Am Geriatr Soc 2003;51:819-823.
16 Shen J, Gao S, Unverzagt FW, Ogunniyi A, Baiyewu O, Gureje O, Hendrie HC, Hall KS: Validation analysis of informant's ratings of cognitive function in African Americans and Nigerians. Int J Geriatr Psychiatry 2006; 21:618-625

17 Tombaugh TN, McIntyre NJ: The MiniMental State Examination: a comprehensive review. J Am Geriatr Soc 1992;40:922-935.

18 Koski L, Xie H, Finch L: Measuring cognition in a geriatric outpatient clinic: Rasch analysis of the Montreal Cognitive Assessment. J Geriatr Psychiatry Neurol 2009;22: 151-160.

$>19$ Nazem S, Siderow AD, Duda JE, Have TT, Colcher A, Horn SS, Moberg PJ, Wilkinson JR, Hurtig HI, Stern MB, Weintraub D: Montreal cognitive assessment performance in patients with Parkinson's disease with 'normal' global cognition according to MiniMental State Examination score. J Am Geriatr Soc 2009;57:304-308

20 Rahman TT, El Gaafary MM: Montreal Cognitive Assessment Arabic version: reliability and validity prevalence of mild cognitive impairment among elderly attending geriatric clubs in Cairo. Geriatr Gerontol Int 2009;9: 54-61. 